\title{
REDUCTION OF A MATRIX DEPENDING ON PARAMETERS TO A DIAGONAL FORM BY ADDITION OPERATIONS
}

\author{
L. N. VASERSTEIN
}

(Communicated by Louis J. Ratliff, Jr.)

\begin{abstract}
It is shown that any $n$ by $n$ matrix with determinant 1 whose entries are real or complex continuous functions on a finite dimensional normal topological space can be reduced to a diagonal form by addition operations if and only if the corresponding homotopy class is trivial, provided that $n \neq 2$ for real-valued functions; moreover, if this is the case, the number of operations can be bounded by a constant depending only on $n$ and the dimension of the space. For real functions and $n=2$, we describe all spaces such that every invertible matrix with trivial homotopy class can be reduced to a diagonal form by addition operations as well as all spaces such that the number of operations is bounded.
\end{abstract}

Introduction. Let $X$ be a topological space $\mathbf{R}^{X}$ the ring of all continuous functions $X \rightarrow \mathbf{R}$ (the reals), $\mathbf{R}_{0}^{X}$ the subring of bounded functions. For any natural number $n$ and a ring $A, M_{n} A$ denotes the ring of all $n$ by $n$ matrices over $A$.

A matrix $\alpha$ in $M_{n} \mathbf{R}^{X}$ can be regarded as a real matrix depending continuously on a parameter which ranges over $X$, or as a continuous map $X \rightarrow M_{n} \mathbf{R}$.

Assume now that $\operatorname{det}(\alpha)=1$, i.e. $\alpha \in \mathrm{SL}_{n} \mathbf{R}^{X}$. We want to reduce $\alpha$ to the identity matrix $1_{n}$ by addition operations, i.e. represent $\alpha$ as a product of elementary matrices $a^{i j}$, where $a \in A=\mathbf{R}^{X}, 1 \leq i \neq j \leq n$. Since the subgroup $E_{n} A$ of $\mathrm{SL}_{n} A$ generated by all elementary matrices is normal [6], it does not matter whether we use row or column addition operations, or both. Note that, by the Whitehead lemma, every diagonal matrix in $\mathrm{SL}_{n} A$ is a product of $4(n-1)$ elementary matrices (for any commutative ring $A$ ), so a matrix $\alpha$ in $\mathrm{SL}_{n} A$, can be reduced to $1_{n}$ if and only if it can be reduced to a diagonal form.

When $X$ is a point, so $A=\mathbf{R}^{X}=\mathbf{R}$, it is well known that this can be done. Moreover [3, Remark 10 with $\operatorname{sr}(\mathbf{R})=m=1]$, this can be done using at most $(n-1)(3 n / 2+1)$ addition operations.

For an arbitrary $X$, a homotopy obstruction may exist which prevents the reduction. Namely, the addition operations do not change the homotopy class $\pi(\alpha)$ of the corresponding map $X \rightarrow \mathrm{SL}_{n} \mathbf{R}$. So if this class is not trivial, the reduction is impossible.

Assume now that the homotopy class $\pi(\alpha)$ is trivial (for example, this is always the case when $X$ is contractible). Is it possible to reduce $\alpha$ to $1_{n}$ by addition operations, i.e. does $\alpha$ belong to the subgroup $E_{n} \mathbf{R}^{X}$ of $\mathrm{SL}_{n} \mathbf{R}^{X}$ generated by elementary matrices)? If yes, how many operations are needed?

Received by the editors March 2, 1987 and, in revised form, June 18, 1987.

1980 Mathematics Subject Classification (1985 Revision). Primary 18F25. 
In this paper, we give an answer to both questions. It turns out that the answer in case $n=2$ is different from that in the case $n \neq 2$. The reason is that the fundamental group $\pi_{1}\left(\mathrm{SL}_{n} \mathbf{R}\right)$ is infinite when $n=2$ (namely, it is infinite cyclic) and it is finite otherwise (it is of order 2 when $n \geq 3$ ).

More precisely, for any $\alpha$ in $\mathrm{SL}_{n} A$ (where $A$ is a commutative ring with 1 such as $A=\mathbf{R}^{X}$ or $\mathbf{R}_{0}^{X}$ ), denote by $l_{A}(\alpha)$ the least $k$ such that $\alpha$ is a product of $k$ elementary matrices over $A$. If no such $k$ exists, i.e. $\alpha$ is outside $E_{n} A$, we set $l_{A}(\alpha)=\infty$. As in [3], $e_{n}(A)$ denotes the supremum of $l_{A}(\alpha)$, where $\alpha$ ranges over $E_{n} A$.

THEOREM 1. Let $X$ be a topological space, $A=\mathbf{R}^{X}$ or $\mathbf{R}_{0}^{X}$ as above. Then (a) $e_{2}(A)<\infty$ if and only if $\mathbf{R}^{Y}=\mathbf{R}$ for every connected component $Y$ of $X$; (b) $l_{A}(\alpha)<\infty$ for all $\alpha$ in $\mathrm{SL}_{2} A$ with $\pi(\alpha)=0$ if and only if $X$ is pseudocompact, i.e. $\mathbf{R}^{X}=\mathbf{R}_{0}^{X}$.

Now we consider the case $n \geq 3$.

THEOREM 2. For any integers $n \geq 3$ and $d \geq 0$ there is a natural number $z$ such that $l_{A}(\alpha) \leq z$ for $A=\mathbf{R}^{X}$ or $\mathbf{R}_{0}^{X}$ with any normal topological space $X$ of dimension $d$ and any $\alpha$ in $\mathrm{SL}_{n} A$ with $\pi(\alpha)=0$. In particular, $e_{n}(A) \leq z$.

As a consequence of Theorem 2 (which is extracted here from results of $[\mathbf{1}, \mathbf{2}]$ ) we obtain that $\mathrm{SL}_{n} A / E_{n} A$ is a homotopy type invariant of $X$ for finite dimensional spaces $X$ if $n \geq 3$. This was proved in [6] for $X=\mathbf{R}$ and in [4] for $X=\mathbf{R}^{3}$ by different methods.

It is easy to extend Theorems 1 and 2 to subrings $A$ of $\mathbf{R}^{X}$ different from $\mathbf{R}^{X}$ and $\mathbf{R}_{0}^{X}$, compare with [6]. This is because of the following fact.

Proposition 3. Let $A$ be as in Theorem 1 and $B$ is a subring with 1 of $A$ such that $B$ is dense in $A$ and $\mathrm{GL}_{1} B$ is open in $B$, both in the topology of uniform convergence. Then $\left|e_{n}(B)-e_{n}(A)\right| \leq(n+3)(n-1)$ for every $n$.

Note that the condition that $\mathrm{GL}_{1} B$ is open in $B$, i.e. $f B=B$ for every function $f$ in $B$ sufficiently close to 1 , cannot be dropped. The following example shows this. Let $X$ be the unit interval $0 \leq x \leq 1$ and $B=\mathbf{R}[x]$, the polynomial ring. In this example, $\mathrm{SL}_{n} B=E_{n} B$ for all $n$, but $e_{n}(B)=\infty$ for each $n \geq 2$ [5]. At the same time, $B$ is dense in $A=\mathbf{R}^{X}=\mathbf{R}_{0}^{X}$ and $e_{n}(A)<\infty$ for $n \geq 3$ by Theorem 1 .

Next we consider the ring $\mathbf{C}^{X}$ of all continuous functions $X \rightarrow \mathbf{C}$, the complex numbers, and its subring $\mathbf{C}_{0}^{X}$ of bounded functions.

THEOREM 4. For any natural number $n$ and an integer $d \geq 0$ there is a natural number $z^{\prime}$ such that $l_{A}(\alpha) \leq z^{\prime}$ for any normal topological space $X$ of dimension $d$ and any matrix $\alpha$ in $\mathrm{SL}_{n} A$ with $\pi(\alpha)=0$, where $A=\mathbf{C}^{X}$ or $\mathbf{C}_{0}^{X}$. In particular, $e_{n}(A) \leq z^{\prime}<\infty$ for all $n$.

COROLLARY 5. For each natural number $n$ and an integer $d \geq 0$ there is a natural number $z^{\prime \prime}$ such that $e_{n}(B) \leq z^{\prime \prime}<\infty$ for any dense subring $B$ with 1 of $A$ with $\mathrm{GL}_{1} B$ open in $B$, where $A$ is as in Theorem 4 .

Note that $\mathbf{C}^{X}$ is endowed with the topology of the uniform convergence, and that the constant $z^{\prime \prime}$ depends only on $n$ and the dimension of $X$. We do not give any 
explicit bounds in this paper, although the proofs in $[1,2]$ seem to be constructive enough to yield some explicit bounds.

ACKNOWLEDGEMENTS. I discussed $\mathrm{SK}_{1}\left(\mathbf{R}^{X}\right)$ and related topics with many mathematicians. Particularly useful were conversations with M. Freedman in February 1987. A. Ocneanu and the referee corrected a few misprints.

Proof of TheOREM 1. Let $X$ be a topological space, $A=\mathbf{R}^{X}$ or $\mathbf{R}_{0}^{X}$ as in Theorem 1. For any $f \in \mathbf{R}^{X}$ we set

$$
\rho f=\left(\begin{array}{cc}
\cos (f) & \sin (f) \\
-\sin (f) & \cos (f)
\end{array}\right) \in \mathrm{SO}_{2} \mathbf{R}^{X}=\mathrm{SO}_{2} \mathbf{R}_{0}^{X} \subset \mathrm{SL}_{2} \mathbf{R}_{0}^{X} .
$$

For any $f \in \mathbf{C}^{X}$, let $\|f\|=\sup |f(x)|$, where $x$ ranges over $X$.

LEMMA 6. Let $\alpha$ be a product of $k$ elementary matrices in $\mathrm{SL}_{2} A$ with $k \geq 1$. Then $\alpha$ has the form $\delta \varepsilon(\rho f)$, where $\varepsilon$ is an elementary matrix, $\delta$ is a diagonal matrix, $f \in \mathbf{R}^{X}$ and $\|f\| \leq(k-1) \pi / 2$.

ProOF. We proceed by induction on $k$. When $k=1$, we can take $\delta=1_{2}$, $f=0$. Assume now that $k \geq 2$ and $\alpha=\varepsilon_{1} \cdots \varepsilon_{k}$ with elementary matrices $\varepsilon_{i}$. By the induction hypothesis, $\varepsilon_{2} \cdots \varepsilon_{k}=\delta^{\prime} \varepsilon^{\prime}\left(\rho\left(f^{\prime}\right)\right)$ with an elementary $\varepsilon^{\prime}$, diagonal $\delta^{\prime}$, and $\left\|f^{\prime}\right\| \leq(k-2) \pi / 2$. If the elementary matrices $\varepsilon_{1}$ and $\varepsilon^{\prime}$ are of the same type, i.e. $\varepsilon_{1} \varepsilon^{\prime}$ is an elementary matrix, then $\alpha=\delta^{\prime}\left(\delta^{\prime-1} \varepsilon_{1} \delta^{\prime} \varepsilon^{\prime}\right)\left(\rho\left(f^{\prime}\right)\right)$ is the required representation, i.e. we can take $\delta=\delta^{\prime}, \varepsilon=\delta^{-1} \varepsilon_{1} \delta \varepsilon^{\prime}, f=f^{\prime}$. Assume now that $\varepsilon^{\prime}, \varepsilon_{1}$ are not of the same type, that is either $\varepsilon_{1} \in A^{1,2}$ and $\varepsilon^{\prime} \in A^{2,1}$, or $\varepsilon_{1} \in A^{2,1}$ and $\varepsilon^{\prime} \in A^{1,2}$. Consider the first case (the second one is similar).

Then $\delta^{\prime-1} \varepsilon_{1} \delta^{\prime} \varepsilon^{\prime}=b^{1,2} c^{2,1}$ with $b$ and $c$ in $A$. Applying the Gram-Schmidt process to the rows of this matrix, we obtain

$$
b^{1,2} c^{2,1}=\left(\begin{array}{cc}
1+b c & b \\
c & 1
\end{array}\right)=\left(\begin{array}{cc}
1 / e & 0 \\
0 & e
\end{array}\right)\left(\begin{array}{cc}
e(1+b c) & e b \\
c / e & 1 / e
\end{array}\right)
$$

with $e=\left(1+c^{2}\right)^{1 / 2} \geq 0$,

$$
\begin{aligned}
\left(\begin{array}{cc}
e(1+b c) & e b \\
c / e & 1 / e
\end{array}\right) & =\left(\begin{array}{cc}
1 & b+c+c b c \\
0 & 1
\end{array}\right)\left(\begin{array}{cc}
1 / e & -c / e \\
c / e & 1 / e
\end{array}\right) \\
& =(b+c+c b c)^{1,2} \rho f^{\prime \prime}
\end{aligned}
$$

with $(c / e, 1 / e)=\left(-\sin \left(f^{\prime \prime}\right), \cos \left(f^{\prime \prime}\right)\right)$ and $\left\|f^{\prime \prime}\right\|<\pi / 2$.

Thus, $\alpha=\varepsilon_{1} \cdots \varepsilon_{k}=\varepsilon_{1} \delta^{\prime} \varepsilon^{\prime}\left(\rho\left(f^{\prime}\right)\right)=\delta^{\prime}\left(\delta^{\prime-1} \varepsilon_{1} \delta^{\prime}\right) \varepsilon^{\prime}\left(\rho f^{\prime}\right)=\delta^{\prime}\left(b^{1,2} c^{2,1}\right) \rho f^{\prime}=$ $\delta \varepsilon \rho f$, where

$$
\delta=\delta^{\prime}\left(\begin{array}{cc}
1 / e & 0 \\
0 & e
\end{array}\right)
$$

is a diagonal matrix, $\varepsilon=(b+c+c b c)^{1,2}$ is an elementary matrix, and $f=f^{\prime}+f^{\prime \prime}$ with $\|f\| \leq\left\|f^{\prime}\right\|+\left\|f^{\prime \prime}\right\| \leq(k-2) \pi / 2+\pi / 2=(k-1) \pi / 2$.

Lemma 6 is proved.

COROLlaRY 7. If $X$ is connected, then for any $g \neq 0$ in $A$ we have

$$
l_{A}(\rho g) \geq(\sup (g)-\inf (g)) / \pi+1 .
$$

PROOF. Suppose that $\rho g$ is a product of $k$ elementary matrices. Then by Lemma $6, \rho g=\delta \varepsilon \rho f$ with diagonal $\delta$, elementary $\varepsilon$, and $\|f\| \leq(k-1) \pi / 2$. It 
follows that $\varepsilon=1_{2}$, and $\delta=1_{2}$ or $-1_{2}$, hence $f-g=2 \pi m$ or $\pi+2 \pi m$ with a continuous function $m: X \rightarrow \mathbf{Z}$ (the integers). Since $X$ is connected, $m$ is a constant. Therefore, $\sup (g)-\inf (g)=\sup (f)-\inf (f) \leq 2\|f\| \leq(k-1) \pi$. Thus, $k \geq 1+(\sup (f)-\inf (f)) / \pi=(\sup (g)-\inf (g)) / \pi+1$. The corollary is proved.

Proposition 8. For any $f$ in $A$, we have $l_{A}(\rho f) \leq 2(\sup (f)-\inf (f)) / \pi+6$.

PROOF. If $f$ is not bounded, there is nothing to prove. So we can assume that $f$ is bounded, i.e. $f \in \mathbf{R}_{0}^{X}$, i.e. $r=\sup (f)-\inf (f)<\infty$. Set $t=(\sup (f)+\inf (f)) / 2$ and write $f=t+(f-t)$, where $t$ means a constant function and $|f-t| \leq r / 2$ everywhere on $X$. We have to write $\rho f$ as a product of $k \leq 2 r / \pi+6$ elementary matrices over $A$. Set $s=[r / \pi+1]$.

We have $\rho f=\rho t(\rho((f-t) / s))^{s}$. Note that $|(f-t) / s| \leq r / 2 s=r / 2([r / \pi+1])<$ $\pi / 2$. So $\cos ((f-t) / s) \in \mathrm{GL}_{1} A$. Therefore $\rho((f-t) / s)$ is a product of two elementary matrices and a diagonal matrix, hence $\rho f$ is a product of $\rho(t)$, a diagonal matrix, and $2 s$ elementary matrices. The product of the constant matrix $\rho t$ and the diagonal matrix has an invertible entry in the first column, so it is the product of at most 4 elementary matrices. Thus, $\rho f$ is the product of $2 s+4 \leq 2 r / \pi+2+4=2 r / \pi+6$ elementary matrices. This proves the proposition.

Now we are prepared to prove Theorem 1. The case of empty $X$ is trivial, so let $X$ be nonempty.

To prove part (a) of Theorem 1, suppose first that $\mathbf{R}^{Y}=\mathbf{R}$ for every connected component $Y$ of $X$. Then $\mathbf{R}^{X}=\mathbf{R}^{X^{\prime}}$ and $\mathbf{R}_{0}^{X}=\mathbf{R}_{0}^{X^{\prime}}$, where $X^{\prime}$ is the discrete set of connected components of $X$. So $e_{2}(A)=e_{2}(\mathbf{R})=4<\infty$.

Suppose now that $\mathbf{R}^{Y} \neq \mathbf{R}$ (or, equivalently $\mathbf{R}_{0}^{Y} \neq \mathbf{R}$ ), for some connected component $Y$ of $X$. We will show that then $e_{2}(B)=\infty$ for $B=\mathbf{R}^{Y}$ and for $B=\mathbf{R}_{0}^{Y}$. This will imply that $e_{2}(A)=\infty$.

Pick a nonconstant function $f$ in $\mathbf{R}^{Y}$. By Corollary 7 applied to $Y$ instead of $X, l_{B}(\rho(f m)) \geq m(\sup (f)-\inf (f)) / \pi+1$ for any natural number $m$. Taking large $m$, we conclude that $e_{2}(B)=\infty$.

To prove Theorem 1(b), consider the exact sequence [6] (see also $[\mathbf{1}, \mathbf{2}]$ )

$$
0 \rightarrow \mathbf{R}^{X} / \mathbf{R}_{0}^{X} \rightarrow \mathrm{SL}_{2} A / E_{2} A \rightarrow \pi^{1}(X) \rightarrow 0 .
$$

The sequence says that $X$ is pseudocompact, if and only if $\mathrm{SL}_{2} A / E_{2} A=\pi^{1}(X)$, i.e. if and only if $\alpha \in E_{2} A$ for every $\alpha$ in $\mathrm{SL}_{2} A$ with $\pi(\alpha)=0$.

PROOF OF THEOREM 2. If the theorem is wrong, then for some $n \geq 3$ and $d \geq 0$ there is a sequence $X(i)$ of normal topological spaces of dimension $d$ and $\alpha(i) \in \mathrm{SL}_{n} A(i)$, where $A(i)=\mathbf{R}^{X(i)}$ or $\mathbf{R}_{0}^{X(i)}$ depending on whether $A=\mathbf{R}^{X}$ or $\mathbf{R}_{0}^{X}$ in the theorem, such that $\pi(\alpha(i))=0$ for all $i$ and $l_{A(i)}(\alpha(i)) \rightarrow \infty$. In the case $A=\mathbf{R}_{0}^{X}$, we can bring each $\alpha(i)$ to $\mathrm{SO}_{n} A(i)$ by $(n+6)(n-1) / 2$ addition operations [6, Lemma 21], so we can assume that $\alpha(i) \in \mathrm{SO}_{n} A(i)$.

We define $X$ to be the disjoint union of all $X(i)$. The matrices $\alpha(i) \in \mathrm{SL}_{n} A(i)$ give a matrix $\alpha$ in $\mathrm{SL}_{n} A$ whose restriction to $X(i)$ is $\alpha(i)$. We have $\pi(\alpha)=0$, i.e. the corresponding map $X \rightarrow \mathrm{SL}_{n} \mathbf{R}$ is homotopic to the trivial map $X \rightarrow 1_{n}$. Now we invoke results of $[\mathbf{1}, \mathbf{2}]$ to conclude that the map $X \rightarrow \mathrm{SL}_{n} \mathbf{R}$ is uniformly homotopic to the trivial map.

First of all, Gram-Schmidt's process [6] reduces the matrix $\alpha$ in $\mathrm{SL}_{n} A$ (as well as its homotopy to the trivial map) to a matrix $f: X \rightarrow \mathrm{SO}_{n} \mathbf{R}$ in the special 
orthogonal group (of the sum of $n$ squares) $\mathrm{SO}_{n} A$ by addition operations (resp. to a homotopy of $f$ to the trivial map into this subgroup). Since $n \geq 3$, the fundamental group $\pi_{1} \mathrm{SO}_{n} \mathbf{R}=\mathbf{Z} / 2 \mathrm{Z}$ is finite. Since $X$ is finite dimensional and normal, Theorem 1 of [1] (see [2] for a shorter and a great deal more transparent proof) gives the desired conclusion.

Thus, $f$ is uniformly homotopic to the trivial map in $\mathrm{SO}_{n} \mathbf{R}$, i.e. the corresponding matrix in $\mathrm{SO}_{n} A$ belongs to the connected component of $1_{n}$, hence $\alpha$ belongs to the connected component of $1_{n}$ in $\mathrm{SL}_{n} A$, where $\mathrm{SL}_{n} A$ is endowed with the topology induced by the uniform convergence topology on $A$.

It is known (see, for example, [6, Theorem 2]) that this component coincides with $E_{n} A$. So $\alpha$ is a product of (finitely many) elementary matrices. Restriction to $X(i)$ yields that each $\alpha(i)$ is the product of a bounded (uniformly in $i$ ) number of elementary matrices over $A(i)$. This contradicts to our choice of $\alpha(i)$ with $l_{A(i)}(\alpha(i)) \rightarrow \infty$. So Theorem 2 is proved.

REMARK. The condition that $X$ is normal can be easily dropped; for arbitrary $X$, the dimension should be understood in the sense of [7], i.e. it is $\operatorname{sr}(A)-1$. It is not clear how $z$ depends on $d$ or whether a uniform upper bound exists. Obviously, $z$ cannot be taken less than $n^{2}-1$, the dimension of $\mathrm{SL}_{n} \mathbf{R}$.

\section{PROOF OF PROPOSITION 3.}

LEMMA 9. Let $B$ be a commutative topological ring with 1 such that $\mathrm{GL}_{1} B$ is open in $B$. Then $l_{B}(\alpha) \leq(n+3)(n-1)$ for any $n$ and any matrix $\alpha$ in $\mathrm{SL}_{n} B$ sufficiently close to $1_{n}$.

PROOF. It is clear that every $\alpha$ sufficiently close to $1_{n}$ has the form $\beta \gamma$ with a lower triangular $\beta$ with ones along the main diagonal and an upper triangular matrix $\gamma$. We have $l_{B}(\beta) \leq n(n-1) / 2$, and $l_{B}(\gamma) \leq(n+6)(n-1) / 2$ by [6, Lemma 21]. So $l_{B}(\alpha) \leq n(n-1) / 2+(n+6)(n-1) / 2=(n+3)(n-1)$.

Let us prove now Proposition 3. Let $\alpha \in E_{n} B$. We can write $\alpha$ as a product of $k=l_{A}(\alpha)$ elementary matrices over $A$. Using that $B$ is dense in $A$ we can write $\alpha$ as a product of $k$ elementary matrices over $B$ and a matrix $\alpha^{\prime}$ arbitrarily close to $1_{n}$. By Lemma $9, \alpha^{\prime}$ is a product of $(n+3)(n-1)$ elementary matrices. So $l_{A}(\alpha) \leq l_{B}(\alpha) \leq l_{A}(\alpha)+(n+3)(n-1)$ for any $\alpha$ in $E_{n} B$. Therefore, $e_{n}(B) \leq$ $e_{n}(A)+(n+3)(n-1)$.

Let now $\alpha \in E_{n} A$. Since $B$ is dense in $A$, we can write $\alpha=\beta \gamma$ with $\beta \in E_{n} B$ and $\gamma$ arbitrarily close to $1_{n}$. So $l_{A}(\alpha) \leq l_{A}(\beta)+(n+3)(n-1)$, by Lemma 9 applied to $A$ instead of $B$. So, $e_{n}(A) \leq e_{n}(B)+(n+3)(n-1)$.

Proposition 3 is proved.

PROOF OF THEOREM 4. If the theorem is wrong, then for some $n \geq 2$ there is a sequence $X(i)$ of normal topological spaces of dimension $d$ and $\alpha(i) \in \mathrm{SL}_{n} A(i)$, where $A(i)=\mathbf{C}^{X(i)}$ or $\mathbf{C}_{0}^{X(i)}$ depending on whether $A=\mathbf{C}^{X}$ or $\mathbf{C}_{0}^{X}$ in the theorem, such that $\pi(\alpha(i))=0$ for all $i$ and $l_{A(i)}(\alpha(i)) \rightarrow \infty$. In the case $A=\mathbf{C}_{0}^{X}$, we can bring each $\alpha(i)$ to $\mathrm{SU}_{n} A(i)$ by $(n+6)(n-1) / 2$ addition operations [6, Lemma 21], so we can assume that $\alpha(i) \in \mathrm{SU}_{n} A(i)$.

We define $X$ to be the disjoint union of all $X(i)$. The matrices $\alpha(i) \in \mathrm{SL}_{n} A(i)$ give a matrix $\alpha$ in $\mathrm{SL}_{n} A$ whose restriction to $X(i)$ is $\alpha(i)$. We have $\pi(\alpha)=0$. By $[1,2], \alpha$ belongs to the connected component of $1_{n}$, where $\mathrm{SL}_{n} A$ is endowed with 
the topology induced by the uniform convergence topology on $A$ (here we used that $\pi_{1}\left(\mathrm{SL}_{n} \mathbf{R}\right)$ is trivial).

It is known that this component coincides with $E_{n} A$. So $\alpha$ is a product of (finitely many) elementary matrices. Restriction to $X(i)$ yields that each $\alpha(i)$ is the product of a bounded (uniformly in $i$ ) number of elementary matrices over $A(i)$. This contradicts our choice of $\alpha(i)$ with $l_{A(i)}(\alpha(i)) \rightarrow \infty$.

So Theorem 4 is proved.

ProOF OF COROLlaRY 5. In the case $A=\mathbf{C}^{X}$, we argue as in the proof of Proposition 3 to conclude that $l_{A}(\alpha) \leq l_{B}(\alpha) \leq l_{A}(\alpha)+(n+3)(n-1)$ for each $\alpha$ in $\mathrm{SL}_{n} B$, hence $e_{n}(B) \leq e_{n}(A)+(n+3)(n-1)$, and that $e_{n}(A) \leq e_{n}(B)+(n+3)(n-1)$. Thus, $\left|e_{n}(B)-e_{n}(A)\right| \leq(n+3)(n-1)$, so we can take $z^{\prime \prime}=z^{\prime}+(n+3)(n-1)$.

\section{REFERENCES}

1. Allan Calder and Jerrold Siegel, Homotopy and uniform homotopy, Trans. Amer. Math. Soc. 235 (1978), 245-269.

2. __ Homotopy and uniform homotopy. II, Proc. Amer. Math. Soc. 78 (1980), 288-290.

3. R. K. Dennis and L. N. Vaserstein, On a question of M. Newman on the number of commutators, J. Algebra (submitted).

4. W. Thurston and L. Vaserstein, On $K_{1}$-theory of the Euclidean space, Topology Appl. 23 (1986), 145-148.

5. W. van der Kallen, $\mathrm{SL}_{3}(\mathbf{C}[x])$ does not have bounded word length, Lecture Notes in Math., vol. 966, Springer-Verlag, Berlin and New York, 1982, pp. 357-362.

6. L. N. Vaserstein, On $K_{1}$-theory of topological spaces, Contemp. Math., vol. 55, Amer. Math. Soc., Providence, R.I., 1986, pp. 729-740.

7. _ The stable range of rings and the dimension of topologcial spaces, Funktsional Anal. i Prilozhen. 5 (1971), 17-27.

Department of Mathematics, Penn State University, University Park, Pennsylvania, 16802 